\title{
Transparent vertex boundary conditions for quantum graphs: Simplified approach
}

\author{
M. M. Aripov ${ }^{1}$, K. K. Sabirov ${ }^{2}$, J. R. Yusupov ${ }^{3}$ \\ ${ }^{1}$ National University of Uzbekistan, Vuzgorodok, Tashkent, Uzbekistan \\ ${ }^{2}$ Tashkent University of Information Technologies, 108 Amir Temur Str., 100200, Tashkent, Uzbekistan \\ ${ }^{3}$ Turin Polytechnic University in Tashkent, 17 Niyazov Str., 100095, Tashkent, Uzbekistan
}

jambul.yusupov@gmail.com

PACS 03.65.Aa, 02.60.Cb, 05.60.Gg

DOI 10.17586/2220-8054-2019-10-5-505-510

\begin{abstract}
We consider quantum graphs providing reflectionless wave transmission at the vertices. Imposing Kuska's version of so-called absorbing boundary conditions we derive the constraints, which make usual continuity and Kirchhoff conditions equivalent to transparent boundary conditions.
\end{abstract}

Keywords: quantum graph, transparent boundary conditions, ballistic transport, wave packet dynamics.

Received: 14 October 2019

Revised: 18 October 2019

\section{Introduction}

The problem of wave propagation in absorbing or transparent media is of importance for different practical applications in optics, acoustics, fluid dynamics and condensed matter physics. An effective way for modeling of wave dynamics in transparent media is describing them in terms of a wave equation, for which so-called transparent boundary conditions are imposed. The problem of wave equation with transparent boundary conditions has attracted much attention in mathematical physics (see, e.g., papers [1-10] for review). Such boundary conditions describe absorption or reflectionless motion of waves in their transmission from one domain to a different one. Therefore, one uses similar terminology for the boundary conditions of both types of processes, calling them absorbing or transparent boundary conditions. The problem of transparent boundary conditions (TBC) for linear partial differential equations (PDEs) was first introduced in the Refs. [1,2] and has become a well developed topic in mathematical and theoretical physics now (see, e.g., [3-18] for review). For both (absorbing and transparent transmission) processes, the boundary conditions can be derived by factorization of the differential operator corresponding to a wave equation, which, in general, leads to complicated equations for the boundary conditions. Explicit form of such boundary conditions are much complicated than those of Dirichlet, Neumann and Robin conditions.

For transparent (absorbing) boundary conditions, the wave equation cannot be solved analytically and always requires using highly accurate numerical methods. In other words, numerical implementation of transparent boundary conditions is a complicated problem, which requires finding effective discretization schemes. Therefore, some authors proposed different simplifications of TBC for the linear Schrödinger equation. Shibata derived such TBC from dispersion relations for the linear Schrödinger equation [19]. Further simplification of TBC obtained by approximating such dispersion relations has been derived by Kuska [20]. Here, we use Kuska's approach for the derivation of simplified vertex boundary conditions for quantum graphs. The latter represent system of quantum wires connected to each other through some rule and are described in terms of the Schrödinger equation on metric graphs [21-25]. Strict mathematical derivation of TBC for quantum graphs has been presented recently in [26]. However, the explicit form of TBC derived in that paper are quite complicated for numerical implementation. Here, we derive a rather simplified version of TBC using the above mentioned Kuska's approach. The explicit form of boundary conditions obtained within such approach is much simpler than those derived in the Ref. [26].

\section{Absorbing boundary conditions on interval}

Following the Ref. [20], we briefly recall the derivation of the approximate transparent boundary conditions for the one-dimensional Schrödinger equation on a finite interval. Consider the one-dimensional time-dependent Schrödinger equation (in the units $m=\hbar=1$ ):

$$
i \frac{\partial \psi}{\partial t}(x, t)=-\frac{1}{2} \frac{\partial^{2} \psi}{\partial x^{2}}(x, t)+V(x) \psi(x, t),
$$


which describes the motion of a quantum particle in the interval $x \in(-\infty,+\infty)$ under the influence of the spacedependent potential $V$. Taking a plane wave of the form

$$
\psi(x, t)=\exp [-i(\omega t-k x)]
$$

from Eqs.(1) and (2), one obtains the dispersion relation for the wave vector $k$, cf. [20]

$$
k^{2}=2[\omega-V] .
$$

This dispersion relation can be solved for $k$ and yields

$$
k= \pm \sqrt{2[\omega-V]},
$$

where the plus sign describes waves moving to $x=+\infty$ and the minus sign means waves moving to $x=-\infty$. The left boundary has to be transparent for left-propagating waves and the right boundary must be transparent for right propagating waves. The main idea used in the Refs. $[19,20]$ for the derivation of absorbing (transparent) boundary conditions was the requirement that plane wave should remain as plane on both sides of the boundary.

The square root function can be approximated using the rational function approximation method following [20]:

$$
\sqrt{z} \approx \frac{1+3 z}{3+z} .
$$

With this approximation (5) for the square root in the dispersion relation (4), one obtains

$$
k= \pm k_{0} \sqrt{\frac{2(\omega-V)}{k_{0}^{2}}} \approx \pm k_{0} \frac{1+3 z}{3+z}, \quad \text { with } \quad z=\frac{2(\omega-V)}{k_{0}^{2}} .
$$

Then, we write (6) as $k(3+z) \approx \pm k_{0}(1+3 z)$ and inserting expression for $z$ into this equation, one obtains:

$$
k\left(3 \frac{k_{0}^{2}}{2}+\omega-V\right) \approx \pm k_{0}\left(\frac{k_{0}^{2}}{2}+3 \omega-3 V\right) .
$$

Now by requiring that the boundaries of a given interval $-L<x<L$ should be transparent (i.e., the boundary does not break profile of the plane wave) and using the correspondence between quantities $k$ and $\omega$ with their operator definitions

we obtain the TBC at the boundaries $x= \pm L$ :

$$
k \leftrightarrow-i \partial / \partial x \text { and } \omega \leftrightarrow i \partial / \partial t
$$

$$
\begin{aligned}
-\left.i\left(3 \frac{k_{0}^{2}}{2}-V\right) \frac{\partial \psi}{\partial x}(x, t)\right|_{x= \pm L} & +\left.\frac{\partial^{2} \psi(x, t)}{\partial x \partial t}\right|_{x= \pm L}= \\
& = \pm\left. k_{0}\left(\frac{k_{0}^{2}}{2}-3 V\right) \psi(x, t)\right|_{x= \pm L} \pm\left. 3 i k_{0} \frac{\partial \psi(x, t)}{\partial t}\right|_{x= \pm L} .
\end{aligned}
$$

Plus and minus signs in Eq.(7) corresponds to the boundary conditions for the right and left walls, respectively. Eq.(1) together with the boundary condition (7) present the problem of absorbing boundary conditions for 1D box.

\section{Absorbing boundary conditions for quantum graph}

In this section, we extend the treatment of the previous section to the case of quantum graphs. To do so, we start from the simplest, star-shaped graph presented in Fig. 1. The Schrödinger equation on such graph can be written as

$$
\frac{\partial \Psi_{j}}{\partial t}(x, t)=\left[-\frac{1}{2} \frac{d^{2}}{d^{2} x}+V_{j}\right] \Psi_{j}(x, t),
$$

where $j=1,2,3$ denotes the bond index, $V_{j}$ is the external potential on $j$ th bond. The coordinates are chosen as $-L_{1}<x<0$ for $j=1$ and $0<x<L_{j}$ for $j=1,2$. In order to solve Eq.(8), one needs to impose boundary conditions at the (internal) vertex and at the edges (external vertices) of each bond. In this work we focus on the internal vertex, therefore the choice of boundary conditions at external vertices are not essential. Here they are imposed as Dirichlet BC, while the vertex boundary conditions are imposed as continuity condition of the wave function weights at the graph vertex:

$$
\left.\alpha_{1} \Psi_{1}(x, t)\right|_{x=0}=\left.\alpha_{2} \Psi_{2}(x, t)\right|_{x=0}=\left.\alpha_{3} \Psi_{3}(x, t)\right|_{x=0},
$$

where $\alpha_{j},(j=1,2,3)$ are the arbitrary constant, which will be determined below. The second boundary condition is imposed as the "current conservation" at the internal vertex

$$
\left.\frac{1}{\alpha_{1}} \frac{d \Psi_{1}(x, t)}{d x}\right|_{x=0}=\left.\frac{1}{\alpha_{2}} \frac{d \Psi_{2}(x, t)}{d x}\right|_{x=0}+\left.\frac{1}{\alpha_{3}} \frac{d \Psi_{3}(x, t)}{d x}\right|_{x=0} .
$$




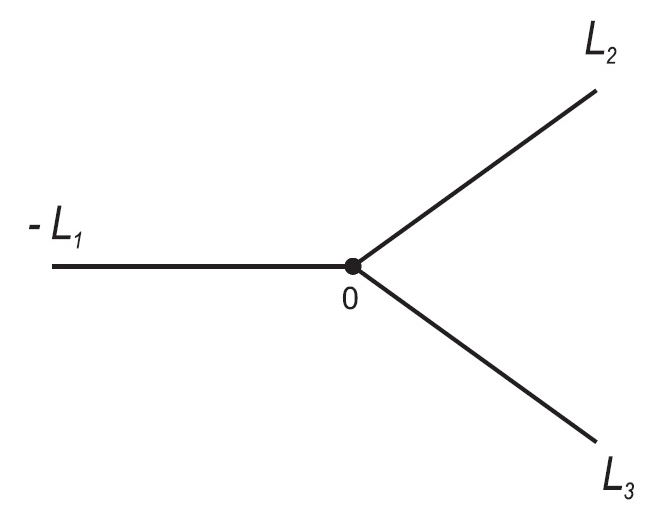

FIG. 1. Sketch of a metric star graph. $L_{j}$ is the length of the $j$ th bond with $j=1,2,3$

Now we apply for such graph prescription for the derivation of absorbing $\mathrm{BC}$ used in the previous section. The dispersion relation for this case can be written as

$$
k_{j}= \pm \sqrt{2\left[\omega_{j}-V_{j}\right]} .
$$

Applying the Eqs.(5) and (11), we derive the absorbing vertex boundary conditions (AVBCs) for quantum graph:

$$
\begin{aligned}
-\left.i\left(3 \frac{k_{0 j}^{2}}{2}-V_{j}\right) \frac{\partial \psi_{j}}{\partial x}(x, t)\right|_{x=0} & \left.\frac{\partial^{2} \psi_{j}(x, t)}{\partial x \partial t}\right|_{x=0}= \\
& -\left.k_{0 j}\left(\frac{k_{0 j}^{2}}{2}-3 V_{j}\right) \psi_{j}(x, t)\right|_{x=0}-\left.3 i k_{0 j} \frac{\partial \psi_{j}(x, t)}{\partial t}\right|_{x=0},
\end{aligned}
$$

Under weak assumptions $k_{0 j}=k_{0}, V_{j}=V_{0}=$ const, from (9) and (10), we get the following constraint

$$
\frac{1}{\alpha_{1}^{2}}=\frac{1}{\alpha_{2}^{2}}+\frac{1}{\alpha_{3}^{2}} .
$$

Eq.(13) is the condition that makes possible fulfilling the boundary conditions given by Eq.(12) with the plane wave solutions of Eq.(8).

It should be noted here that for other two choices, when the second or third bonds are incoming, such constraints can be derived analogously. Thus, for the incoming second bond and outgoing first and third bonds, the constraint is

$$
\frac{1}{\alpha_{2}^{2}}=\frac{1}{\alpha_{1}^{2}}+\frac{1}{\alpha_{3}^{2}},
$$

and for the incoming third bond and outgoing first and second bonds the constraint is

$$
\frac{1}{\alpha_{3}^{2}}=\frac{1}{\alpha_{1}^{2}}+\frac{1}{\alpha_{2}^{2}} \text {. }
$$

\section{Numerical experiment}

In this section, we show numerically that fulfilling the constraint (13) allows reflectionless transmission of the Gaussian wave packet (GWP) through the vertex (branching point) of a graph with boundary conditions in the form of (9) and (10). The configuration of the experimental set-up consists of star graph with three bonds (see Fig. 1) of lengths $L_{1}=L_{2}=L_{3}=10$. We consider the wave going from the first bond to the second and the third ones, i.e., the initial condition is compactly supported in the first bond. The initial state of the wave function is given by the following Gaussian function

$$
\Psi^{I}(x)=(2 \pi)^{-1 / 4} \exp \left(5 i x-(x-5)^{2} / 4\right)
$$

To solve Eq.(8) numerically, we use Crank-Nicolson finite difference scheme with the space discretization $\Delta x=$ 0.016 and the time step $\Delta t=5 \cdot 10^{-5}$. We consider time interval $[0,1.1]$, which is enough for complete splitting of GWP into the second and the third bonds.

Firstly, we simulate GWP dynamics in a quantum star graph with "natural" vertex boundary conditions, i.e. boundary conditions given by (9) and (10) with $\alpha_{1}=\alpha_{2}=\alpha_{3}=1$. For this case we have reflection of GWP with "mass" of fraction $N_{1}=1 / 9$ (see, Ref. [21]). Other $8 / 9$ part of mass is transmits through the vertex and splits into 

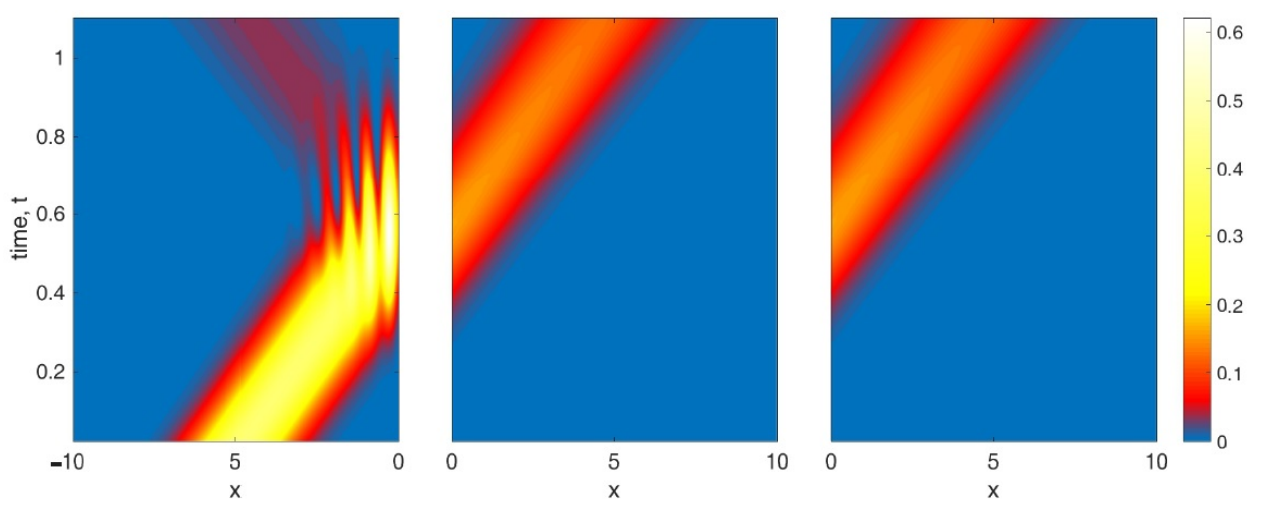

FIG. 2. Contour plot of the probability density for the GWP initially located on the first bond and given by (14). The imposed vertex boundary conditions are "natural" ones, i.e. $\alpha_{1}=\alpha_{2}=\alpha_{3}=1$. Each column corresponds to a bond, numbering from left to right

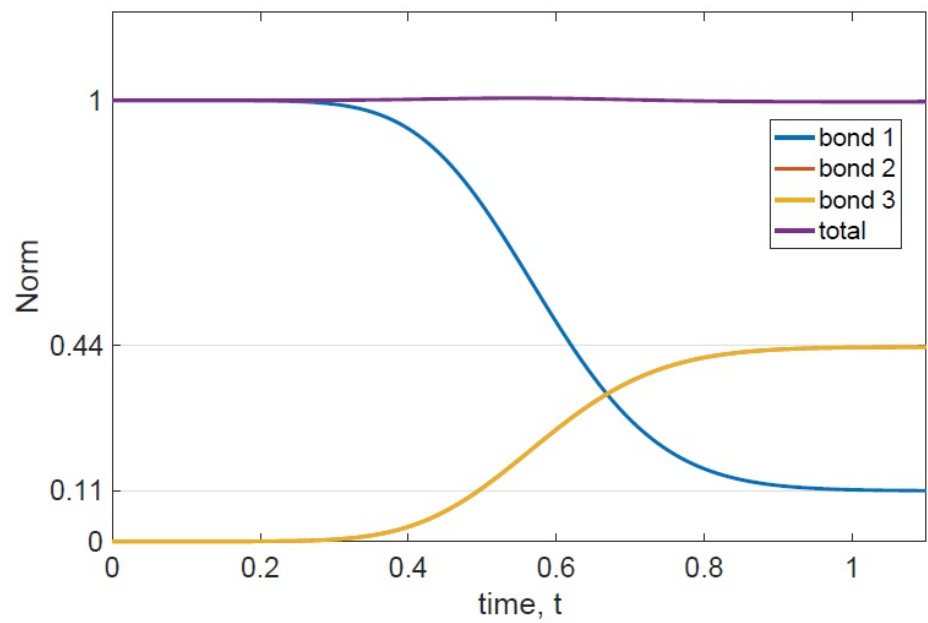

FIG. 3. Partial norms of GWP for the case shown in Fig. 2
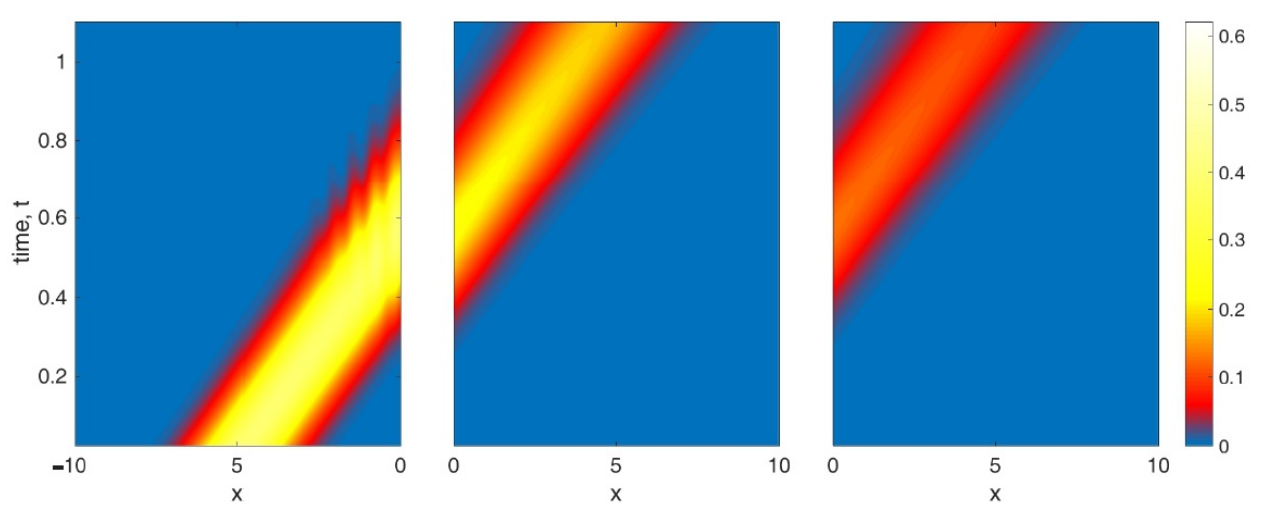

FIG. 4. Contour plot of the probability density for the GWP initially located on the first bond and given by (14). The imposed vertex boundary conditions are Kirchhoff-type ones with $\alpha_{1}=2.4$, $\alpha_{2}=3$ and $\alpha_{3}=4$. Each column corresponds to a bond, numbering from left to right 


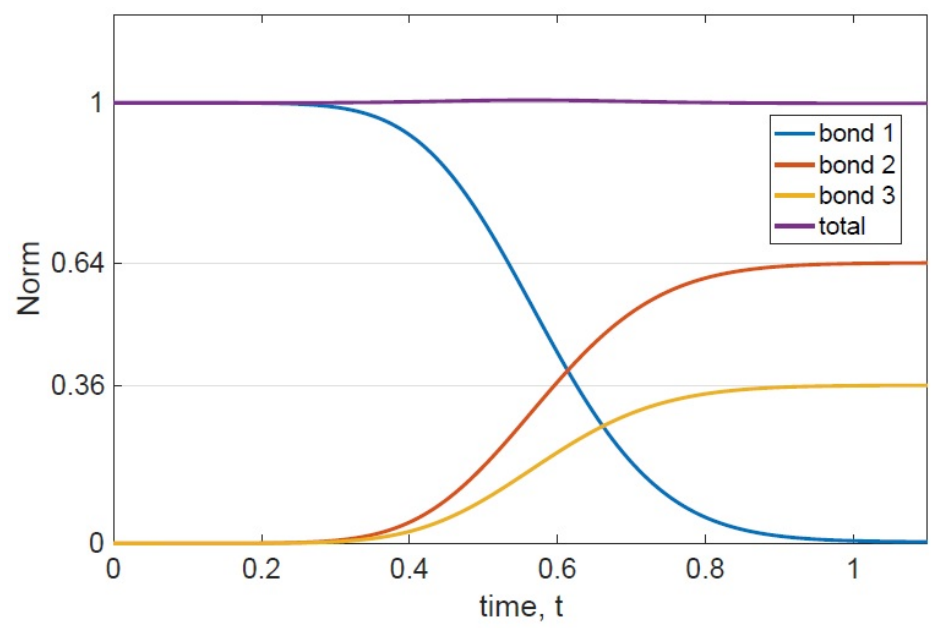

FIG. 5. Partial norms of the GWP for the case shown in Fig. 4. The masses of transmitted fractions are $N_{2}=0.64$ and $N_{3}=0.36$, which confirm $N_{j}=\alpha_{j}^{2} /\left(\alpha_{2}^{2}+\alpha_{3}^{2}\right), j=1,2$

two identical fractions. Fig. 2 demonstrates this dynamics and in the Fig. 3 one can see the time-dependence of partial norms.

Now, we choose parameters $\alpha_{j}$ so that they fulfill the constraint (13): $\alpha_{1}=2.4, \alpha_{2}=3$ and $\alpha_{3}=4$. The GWP dynamics for this set-up is shown in Fig. 4 and Fig. 5. Here we note that it is easy to show the possibility of controlling of the GWP transition through the vertex by choosing proper parameters $\alpha_{2,3}$ so that the "masses" of fractions will be $N_{2}=\alpha_{2}^{2} /\left(\alpha_{2}^{2}+\alpha_{3}^{2}\right)$ and $N_{3}=\alpha_{3}^{2} /\left(\alpha_{2}^{2}+\alpha_{3}^{2}\right)$, accordingly.

\section{Conclusions}

We have studied reflectionless wave transport in quantum graphs using simplified version of transparent boundary conditions concept (so-called Kuska's approach). It is shown that within such simplification, under certain constraints, usual continuity condition and Kirchhoff rules imposed at the graph vertex become equivalent to transparent boundary conditions. Reflectionless transmission of quantum particle through the graph branching point is shown by numerical solution of the time-dependent Schrödinger equation on a graph. Although the problem is solved for simple star graph, extension of the approach for other graph (branching) topologies is rather trivial.

\section{References}

[1] Engquist B., Majda A. Absorbing boundary conditions for the numerical simulation of waves. Math. Comput., 1977,31 , P. 629-651.

[2] Kosloff R., Kosloff D., Absorbing boundaries for wave propagation problems. J. Comput. Phys., 1986, 63(2), P. $363-376$.

[3] Arnold A., Ehrhardt M. Discrete Transparent Boundary Conditions for Wide Angle Parabolic Equations in Underwater Acoustics. J. Comput. Phys., 1998, 145(2), P. 611-638.

[4] Ehrhardt M. Discrete Transparent Boundary Conditions for General Schrödinger-type Equations. VLSI Design, 1999, 9(4), P. 325-338.

[5] Ehrhardt M., Arnold A. Discrete transparent boundary conditions for the Schrödinger equation. Riv. di Math. Univ. di Parma, 2001, 6(4), P. 57-108.

[6] Ehrhardt M. Efficient Numerical Implementation of Discrete Transparent Boundary Conditions for Parabolic Equations in Underwater Acoustics. Acta Acustica united with Acustica, 2002, 88, P. 711-713.

[7] Arnold A., Ehrhardt M. and Sofronov I. Discrete transparent boundary conditions for the Schrdinger equation: fast calculation, approximation, and stability. Comm. Math. Sci., 2003, 1(3), P. 501-556.

[8] Jiang S. and Greengard L. Fast evaluation of nonreflecting boundary conditions for the Schrödinger equation in one dimension. Comput. Math. Appl., 2004, 47, P. 955-966.

[9] Antoine X., Arnold A., Besse C., Ehrhardt M. and Schädle A., A Review of artificial boundary conditions for the Schrödinger equation. Commun. Comput. Phys., 2008, 4(4), P. 729-796.

[10] Ehrhardt M. Discrete transparent boundary conditions for Schrd̈inger-type equations for non-compactly supported initial data. Appl. Numer. Math., 2008, 58(5), P. 660-673.

[11] S̆umichrast L. and Ehrhardt M. Comparison of the continuous, semi-discrete and fully-discrete transparent boundary conditions (TBC) for the parabolic wave equation: 1 theory. J. Electr. Engineering, 2009, 60(6), P. 301-312.

[12] Antoine X., Besse C., Klein P. Absorbing boundary conditions for the one-dimensional Schrödinger equation with an exterior repulsive potential. J. Comput. Phys., 2009, 228(2), P. 312-335.

[13] Ehrhardt M. Absorbing Boundary Conditions for Hyperbolic Systems. Numer. Math.: Theor. Meth. Appl., 2010, 3(3), P. $295-337$.

[14] Klein P., Antoine X., Besse C. and Ehrhardt M. Absorbing Boundary Conditions for Solving N-Dimensional Stationary Schrödinger Equations with Unbounded Potentials and Nonlinearities. Commun. Comput. Phys., 2011, 10(5), P. 1280-1304. 
[15] Arnold A., Ehrhardt M., Schulte M. and Sofronov I. Discrete transparent boundary conditions for the Schrödinger equation on circular domains. Commun. Math. Sci., 2012, 10(3), P. 889-916.

[16] Feshchenko R.M. and Popov A.V. Exact transparent boundary condition for the three-dimensional Schrödinger equation in a rectangular cuboid computational domain. Phys. Rev. E, 2013, 88, P. 053308.

[17] Antoine X. et al., Absorbing boundary conditions for relativistic quantum mechanics equations. J. Comput. Phys., 2014, 277, P. $268-304$.

[18] Petrov P. and Ehrhardt M. Transparent boundary conditions for iterative high-order parabolic equations. J. Comput. Phys., 2016, 313, P. 144158.

[19] Shibata T. Absorbing boundary conditions for the finite-difference time-domain calculation of the one-dimensional Schrödinger equation. Phys. Rev. B, 1991, 43(8), P. 6760.

[20] Kuska J.-P. Absorbing boundary conditions for the Schrödinger equation on finite intervals. Phys. Rev. B, 1992, 46(8), P. 5000.

[21] Kottos T. and Smilansky U. Periodic Orbit Theory and Spectral Statistics for Quantum Graphs. Ann. Phys., 1999,76, P. 274.

[22] Gnutzmann S. and Smilansky U. Quantum graphs: Applications to quantum chaos and universal spectral statistics. Adv. Phys., 2006, 55, P. 527-625. .

[23] Gnutzmann S., Schanz H. and Smilansky U. Topological Resonances in Scattering on Networks (Graphs). Phys. Rev. Lett., 2013, 110, P. 094101.

[24] Gnutzmann S., Waltner D. Stationary waves on nonlinear quantum graphs: General framework and canonical perturbation theory. Phys. Rev. E, 2016, 93, P. 032204.

[25] Gnutzmann S., Waltner D. Stationary waves on nonlinear quantum graphs. II. Application of canonical perturbation theory in basic graph structures. Phys. Rev. E, 2016, 94, P. 062216.

[26] Yusupov J.R., Sabirov K.K., Ehrhardt M. and Matrasulov D.U. Transparent quantum graphs. Phys. Lett. A, 2019, 383, P. $2382-2388$.

[27] Crank J. and Nicolson P. A practical method for numerical evaluation of solutions of partial differential equations of the heat-conduction type. Advances in Comput. Math., 1996, 6(1), P. 207-226.

[28] Dubeibe F.L. Solving the time-dependent Schrödinger equation with absorbing boundary conditions and source terms in Mathematica 6.0. Int. J. Mod. Phys. C, 2010, 21(11), P. 1391-1406.

[29] Jiang Sh. and Greengard L. Fast evaluation of nonreflecting boundary conditions for the Schrödinger equation in one dimension. Computers \& Mathematics with Applications, 2004, 47(6), P. 955-966.

[30] Neuhauser D. and Baer M. The time-dependent Schrödinger equation: Application of absorbing boundary conditions. J. Chem. Phys., 1989, 90(8), P. 4351-4355. 